\title{
Effect of bosentan is correlated with MMP-9/TIMP-1 ratio in bleomycin-induced pulmonary fibrosis
}

\author{
WAN-LI ZUO ${ }^{1}$, JIE-MIN ZHAO ${ }^{1}$, JI-XIONG HUANG ${ }^{1}$, WEI ZHOU ${ }^{2}$, \\ ZE-HONG LEI ${ }^{3}$, YAN-MING HUANG ${ }^{1}$, YAN-FEN HUANG ${ }^{1}$ and HAI-GANG LI ${ }^{4,5}$
}

\begin{abstract}
Departments of ${ }^{1}$ Respiratory Medicine, ${ }^{2}$ Pathology and ${ }^{3}$ Medical Laboratory, The Jiangmen Central Hospital, The Jiangmen Affiliated Hospital of Sun Yat-sen University, Jiangmen, Guangdong 529030; ${ }^{4}$ Department of Pathology, Zengcheng People's Hospital, Guangzhou, Guangdong 511300; ${ }^{5}$ Department of Pathology, Sun Yat-sen Memorial Hospital, Sun Yat-sen University, Guangzhou, Guangdong 510120, P.R. China
\end{abstract}

Received June 15, 2016; Accepted September 28, 2016

DOI: $10.3892 /$ br.2016.832

\begin{abstract}
Pulmonary fibrosis (PF) is a life-threatening non-tumorous disease characterized by progressive fibrosis and worsening lung function. Various drugs, such as bleomycin, can contribute to lung injury and $\mathrm{PF}$, with lung injury potentially occurring in $10 \%$ of bleomycin users. Bleomycin is the most commonly used drug in the establishment of an animal model of PF in rats. Matrix metalloproteinases (MMPs) and tissue inhibitors of metalloproteinases (TIMPs) serve an important role in controlling tissue organization and fibrosis following injury. The present study examined the effect of bosentan on fibrotic lung tissue in rats administrated with bleomycin. In total, 48 Wistar rats were administrated with bleomycin, with or without bosentan, while the control rats received saline. The lung tissues were microscopically examined by staining with hematoxylin and eosin and Masson's trichome. ELISA was also used to detect the MMP-9 and TIMP-1 concentrations in the plasma. The results indicated that the bosentan-treated groups on the next day and the 15th day showed significant reversal of pathological findings. In addition, the concentrations of MMP-9 and TIMP-1 appeared to be altered following bosentan treatment, improving the bleomycin-induced PF. Masson's trichome staining showed high collagen deposition in the lung tissue sections, which may be a direct result of the activity of MMP-9 and TIMP-1. Furthermore, the deposition of collagen was significantly inhibited in bosentan-treated groups. In conclusion, these results demonstrated that bosentan
\end{abstract}

Correspondence to: Professor Hai-Gang Li, Department of Pathology, Sun Yat-sen Memorial Hospital, Sun Yat-sen University, 107 Yanjiang West Road, Guangzhou, Guangdong 510120, P.R. China

E-mail: 13728089120@126.com

Key words: pulmonary fibrosis, matrix metalloproteinase-9, tissue matrix metalloproteinase inhibitor-1, bleomycin inhibited lung fibrosis induced by bleomycin and it may be used as an inhibitor of PF.

\section{Introduction}

Pulmonary fibrosis $(\mathrm{PF})$ is a type of diffuse parenchymal lung disease characterized by chronic inflammation and progressive parenchymal fibrosis (1). PF with unclear etiology is known as idiopathic PF (IPF). The incidence of IPF increases worldwide and is similar to that of certain types of cancer, including stomach and liver cancer (2-4). IPF results in deteriorated lung function and ultimately leads to respiratory failure. Patients with IPF have a poor prognosis, with a median survival of 3 years, which is worse than certain types of cancer (5). The diagnosis of IPF is established after excluding specific known causes of PF and a pattern so-called usual interstitial pneumonia (6).

Numerous drugs, such as bleomycin, are known to contribute to lung injury and PF. Bleomycin is a chemotherapeutic drug, with lung injury and dose-independent PF observed in $10 \%$ of bleomycin users. Bleomycin is commonly used to establish an animal model of PF $(7,8)$. Microscopically, lung injury is followed by inflammation, excessive accumulation and deposition of extracellular matrix (ECM) resulting in the thickening of alveolar interval. Matrix metalloproteinases (MMPs) and tissue matrix metalloproteinase inhibitors (TIMPs) are the main proteases involved in the degradation and remolding of the ECM (9). As a non-selective dual endothelin-1 receptor antagonist, bosentan protects against acute lung injury resulting from PF by reducing inflammation factor release (10). Bosentan also improves PF induced by bleomycin and reduces the expression levels of MMP-2 gene (11). Although both MMP-2 and MMP-9 are gelatinases, only MMP-9 has been shown to be correlated with the degree of bleomycin-induced PF, while its serum levels decrease in patients with pulmonary arterial hypertension and increase following treatment with bosentan (12). However, the roles of MMP-9 and TIMP1 in bosentan treatment for PF have not been studied. Therefore, the aim of the present study was to investigate the effects of bosentan on MMP-9 and TIMP1 expression in a model of bleomycin-induced $\mathrm{PF}$ in rats. 


\section{Materials and methods}

Ethical approval. All animal studies and procedures were approved by the Institutional Animal Use and Care Committee of Sun Yat-sen University (Guangzhou, China; animal welfare assurance no. SCXK 2011-0029; experiment welfare assurance no. SYXK 2012-0081). All rats were maintained according to institutional guidelines. Animal procedures were performed in compliance with the Institutional Standards for Humane Care and Use of Laboratory Animals.

Animals. A total of 48 specific pathogen-free, healthy young rats, aged 7 weeks (weight range, 182-248 g) were obtained from the Center of Experimental Animal of Sun Yat-sen University, and raised in a room with windows (temperature at $22 \pm 20^{\circ} \mathrm{C}$, humidity $55 \pm 5 \%$, natural light/dark, food and water no limited). All rats were randomly assigned to the following groups: Saline (control; C groups), bleomycin-induced PF (F groups) and bleomycin-induced $\mathrm{PF}$ followed by bosentan treatment (B groups). The C, $\mathrm{F}$ and $\mathrm{B}$ groups were also randomly treated for two different time durations prior to sacrifice, including rats treated for 2 weeks (namely groups $\mathrm{C} 1, \mathrm{~F} 1$ and B1) and for 4 weeks (namely groups $\mathrm{C} 2, \mathrm{~F} 2$ and B2). All rats were housed in a specific pathogen-free facility and screened regularly for pathogens. Bleomycin-induced PF rats were anesthetized by isoflurane inhalation prior to treating with intranasal injection of $50 \mathrm{ml}$ sterile saline solution containing $6 \mathrm{U} / \mathrm{kg}$ body weight (7.5 mg/kg body weight) bleomycin (Nippon Kayaku, Tokyo, Japan) (13). Rats in the B groups were then treated with bosentan on the next day (B1) or 15th day (B2) after bleomycin treatment. Control rats $(\mathrm{C} 1$ and $\mathrm{C} 2)$ received a single intratracheal dose of sterile saline alone. The rats were monitored and weighed daily for the duration of the experiment. Animals were euthanized by $\mathrm{CO}_{2}$ asphyxiation at the indicated time-points.

Blood collection. Blood was collected by inferior vena cava puncture following euthanasia. After standing at room temperature for $30 \mathrm{mins}$, the blood was centrifuged for $5 \mathrm{~min}$ at $1,358.37 \mathrm{x}$ at $4^{\circ} \mathrm{C}$. The supernatants were collected and stored at $-20^{\circ} \mathrm{C}$ until further use.

Histopathological analysis of lung tissue. The lungs of rats were perfused with $10 \mathrm{ml}$ cold phosphate-buffered saline before sacrifice (C1, F1, B1 group rats after 2 weeks of treatment, and C2, F2, B2 group rats after 4 weeks of treatment) and then harvested. The right lung lobes were placed in $10 \%$ formalin and embedded in paraffin for sectioning $(25 \times 25 \mathrm{~mm})$. Following deparaffinization, rehydration and washing in deionized water, the slides containing serial sections were stained with H\&E or Masson's trichrome according to routine procedures (14).

Alveolitis and fibrosis were individually assessed by lung histology using the semiquantitative grading system described by Szapiel et al (15): 0, no alveolitis/fibrosis was observed; 1 (mild), focal lesions occupying $<25 \%$ or $<20 \%$ (for alveolitis and fibrosis, respectively) of the lung were detected in the alveolar septum; 2 (moderate), widespread alveolitis or fibrosis involving $25-50 \%$ or $20-50 \%$, respectively, of the lung was observed; and 3 (severe), a diffused alveolitis or fibrosis
Table I. Efficacy of bosentan on body weight of bleomycin-induced fibrosis rats.

\begin{tabular}{lc}
\hline Group & Body weight increase, $g$ \\
\hline C1 & $110.38 \pm 28.32^{\mathrm{a}}$ \\
C2 & $100.50 \pm 16.02^{\mathrm{b}}$ \\
F1 & $22.75 \pm 5.82^{\mathrm{c}}$ \\
F2 & $61.25 \pm 18.68^{\mathrm{d}}$ \\
B1 & $99.63 \pm 22.15^{\mathrm{e}}$ \\
B2 & $57.25 \pm 19.61$ \\
\hline
\end{tabular}

Values are presented as means \pm standard deviation ( $\mathrm{n}=8$ /group). ${ }^{\mathrm{a}} \mathrm{P}<0.05$ vs. $\mathrm{F} 1, \mathrm{~F} 2$ and $\mathrm{B} 2$; ${ }^{\mathrm{b}} \mathrm{P}<0.05$ vs. $\mathrm{F} 1, \mathrm{~F} 2$ and $\mathrm{B} 2$; ${ }^{\mathrm{c}} \mathrm{P}<0.05$ vs. $\mathrm{F} 2$, $\mathrm{B} 1$ and $\mathrm{B} 2 ;{ }^{\mathrm{d}} \mathrm{P}<0.05$ vs. $\mathrm{B} 1 ;{ }^{\mathrm{e}} \mathrm{P}<0.05$ vs. $\mathrm{B} 2$.

spanning $>50 \%$ of the lung was observed, with occasional consolidation of air spaces and patches of hemorrhagic areas within the interstitium. The entire lung section was reviewed under a lower power field (Olympus, BX-51; magnification, $\mathrm{x} 100)$. In total, 20 random microscopic fields per section were examined, and a score ranging between 0 and 3 was assigned. All assessments were performed in double-blind manner.

MMP-9 and TIMP-1 concentrations determined by ELISA. The concentrations of MMP-9 (e02m0329) and TIMP-1 (e02t0047) in the plasma of rats in the various groups were measured by ELISA kits (Shanghai BlueGene Biotech Co., Ltd., Shanghai, China) according to the manufacturer's instructions.

Statistical analysis. Evaluation of statistically significant differences between the groups was performed using Student's t-test and analysis of variance. Data are expressed as the mean \pm standard deviation, as indicated. All analyses of data were performed using SPSS software for Windows (version 13.0; SPSS, Inc., Chicago, IL, USA) and a $\mathrm{P}<0.05$ was considered to indicate a statistically significant difference.

\section{Results}

Changes in body weight. Table I shows the effect of bosentan on the body weight of bleomycin-administered groups of rats. Compared with the normal control rats $(\mathrm{C} 1$ and $\mathrm{C} 2)$, rats administered bleomycin alone (F1 and F2) or along with bosentan treatment after 15 days (B2) had a lower increase in body weight between the beginning of the experiments and sacrifice $(\mathrm{C} 1$ vs. $\mathrm{F} 2$ and $\mathrm{B} 2 ; \mathrm{P}=0.001$, others $\mathrm{P}<0.0001)$. By contrast, the group treated with bosentan on the day following bleomycin administration (B1) demonstrated a similar increase in body weight to the control groups (C1 and $\mathrm{C} 2$ ), and a significantly higher body weight increase when compared with the $\mathrm{F} 1, \mathrm{~F} 2$ and $\mathrm{B} 2$ groups (B1 vs. $\mathrm{C} 1$, $\mathrm{P}=0.706$; $\mathrm{B} 1$ vs. $\mathrm{C} 2, \mathrm{P}=0.858$; $\mathrm{B} 1$ vs. $\mathrm{F} 1, \mathrm{P}<0.0001$; $\mathrm{B} 1$ vs. $\mathrm{F} 2$, $\mathrm{P}=0.003$; and $\mathrm{B} 1$ vs. $\mathrm{B} 2, \mathrm{P}=0.002$ ). However, the body weight of $\mathrm{B} 2$ rats showed no significant change compared with the bleomycin-administrated groups without bosentan treatment $(\mathrm{F} 2 ; \mathrm{P}=0.682)$. 
Table II. Efficacy of bosentan on bleomycin-induced alveolitis and fibrosis rats.

\begin{tabular}{lcc}
\hline Group & Grading of alveolitis & Grading of fibrosis \\
\hline $\mathrm{C} 1^{\mathrm{a}}$ & $0.1 \pm 0.4$ & $0.0 \pm 0.0$ \\
$\mathrm{C} 2^{\mathrm{b}}$ & $0.0 \pm 0.0$ & $0.0 \pm 0.0$ \\
$\mathrm{~F} 1^{\mathrm{c}}$ & $2.6 \pm 0.5$ & $1.4 \pm 0.5$ \\
$\mathrm{~F} 2^{\mathrm{d}}$ & $2.3 \pm 0.7$ & $2.8 \pm 0.5$ \\
$\mathrm{~B} 1^{\mathrm{e}}$ & $1.5 \pm 0.8$ & $1.8 \pm 0.7$ \\
$\mathrm{~B} 2$ & $2.0 \pm 0.8$ & $2.6 \pm 0.5$ \\
\hline
\end{tabular}

Values are presented as means \pm standard deviation ( $n=8 /$ group). Alveolitis and fibrosis, ${ }^{a} \mathrm{P}<0.05$ vs. F1, F2, B1 and B2; ${ }^{b} \mathrm{P}<0.05$ vs. F1, F2, B1 and B2; alveolitis, ${ }^{\mathrm{C}} \mathrm{P}<0.05$ vs. $\mathrm{B} 1$; fibrosis, ${ }^{\mathrm{C}} \mathrm{P}<0.05$ vs. $\mathrm{F} 2$ and $\mathrm{B} 2 ;{ }^{\mathrm{d}} \mathrm{P}<0.05$ vs. $\mathrm{B} 1$ and $\mathrm{B} 2 ;{ }^{\mathrm{e}} \mathrm{P}<0.05$ vs. $\mathrm{B} 2$.

Bosentan attenuates bleomycin-induced alveolitis and lung fibrosis. Microscopically, the histopathological changes in the lung tissues between the control ( $\mathrm{C}$ groups), bleomycin-induced PF (F groups) and bleomycin-induced PF treated with bosentan (B groups) rats were evaluated. The results demonstrated that bleomycin-induced alveolitis and PF were inhibited by bosentan treatment on the next day after bleomycin (Table II). Sections of the control groups had a normal structure without alveolitis and PF (Fig. 1A and B). The most severe alveolitis was identified in group F1, with the lung sections showing infiltration of numerous inflammatory cells, including neutrophilic granulocytes, lymphocytes and macrophages, in the interstitial lung and alveoli. Interstitial edema, diffuse hemorrhage and thickened alveolus interstitium were also observed in the F1 group tissues. In addition, the most significant inhibition of alveolitis was demonstrated when bosentan was administered on the day after bleomycin (B1), which suggested that early administration of bosentan had a better effect than later administration (as in B2). The most severe fibrosis was observed in group F2; those sections showed a thickened alveolus interstitium, and accumulation of collagen and fibrosis, with reduced number of inflammatory cells. Therefore, bosentan treatment for $\mathrm{PF}$ on the following day from bleomycin administration in rats may assist in preventing certain pathological changes induced by bleomycin. Furthermore, reduced inflammatory cell infiltration and collagen deposition was detected in B1 group. However, no significant reduction in alveolitis, lung fibrosis and collagen fibers were observed in group B2, which suggested that bosentan was less effective if administered at a later stage after bleomycin (Fig. 1; Table II).

Effect of bosentan treatmenton expressionlevels of MMP-9 and TIMP-1 in PF lung tissues. The control groups demonstrated the highest concentration of MMP-9 and MMP-9/TIMP-1 ratio, and the lowest concentration of TIMP-1 in the blood serum of rats. However, the concentration of MMP-9 was significantly reduced in all the bleomycin-induced PF groups treated with or without bosentan compared with the control rats $(\mathrm{P}<0.05$; Table III). In addition, the concentration of TIMP-1 in all the bleomycin-induced PF groups treated with or without
Table III. Efficacy of bosentan on bleomycin-induced MMP-9 and TIMP-1 of rats.

\begin{tabular}{lccc}
\hline Group & MMP-9 $(\mu \mathrm{g} / \mathrm{l})$ & TIMP-1 $(\mu \mathrm{g} / \mathrm{l})$ & MMP-9/TIMP-1 \\
\hline $\mathrm{C} 1^{\mathrm{a}}$ & $4.14 \pm 0.86$ & $0.35 \pm 0.16$ & $14.34 \pm 8.67$ \\
$\mathrm{C}^{\mathrm{b}}$ & $4.39 \pm 0.75$ & $0.32 \pm 0.10$ & $14.72 \pm 4.02$ \\
$\mathrm{~F}^{\mathrm{c}}$ & $1.57 \pm 0.85$ & $0.70 \pm 0.08$ & $2.29 \pm 1.29$ \\
$\mathrm{~F}^{\mathrm{d}}$ & $0.29 \pm 0.08$ & $1.18 \pm 0.51$ & $0.28 \pm 0.12$ \\
$\mathrm{~B} 1^{\mathrm{e}}$ & $1.35 \pm 0.55$ & $0.48 \pm 0.16$ & $3.11 \pm 1.58$ \\
$\mathrm{~B} 2$ & $0.60 \pm 0.20$ & $0.75 \pm 0.18$ & $0.88 \pm 0.42$ \\
\hline
\end{tabular}

Values are presented as means \pm standard deviation ( $n=8 /$ group). MMP-9, ${ }^{\mathrm{a}} \mathrm{P}<0.05$ vs. F1, F2, B1 and B2; ${ }^{\mathrm{b}} \mathrm{P}<0.05$ vs. F1, F2, B1 and $\mathrm{B} 2$; ${ }^{\mathrm{C}} \mathrm{P}<0.05$ vs. $\mathrm{F} 2$ and $\mathrm{B} 2 ;{ }^{\mathrm{d}} \mathrm{P}<0.05$ vs. $\mathrm{B} 1$ and $\mathrm{B} 2$; ${ }^{\mathrm{e}} \mathrm{P}<0.05$ vs. B2; TIMP- $1,{ }^{\mathrm{a}} \mathrm{P}<0.05$ vs. F1, F2, B 1 and $\mathrm{B} 2$; ${ }^{\mathrm{b}} \mathrm{P}<0.05$ vs. F1, F2, $\mathrm{B} 1$ and $\mathrm{B} 2 ;{ }^{\mathrm{C}} \mathrm{P}<0.05$ vs. $\mathrm{F} 2$ and $\mathrm{B} 1 ;{ }^{\mathrm{d}} \mathrm{P}<0.05$ vs. $\mathrm{B} 1 ;{ }^{\mathrm{e}} \mathrm{P}<0.05$ vs. $\mathrm{B} 2$; MMP-9/TIMP-1, ${ }^{\mathrm{a}} \mathrm{P}<0.05$ vs. F1, F2, $\mathrm{B} 1$ and $\mathrm{B} 2$; ${ }^{\mathrm{b}} \mathrm{P}<0.05$ vs. $\mathrm{F} 1, \mathrm{~F} 2$, $\mathrm{B} 1$ and $\mathrm{B} 2$; ${ }^{\mathrm{P}}<0.05$ vs. $\mathrm{F} 2$ and $\mathrm{B} 2$; ${ }^{\mathrm{d}} \mathrm{P}<0.05$ vs. $\mathrm{B} 1$ and $\mathrm{B} 2 ;{ }^{\mathrm{e}} \mathrm{P}<0.05$ vs. B2. MMP, matrix metalloproteinases; TIMP, tissue inhibitors of metalloproteinases.

bosentan was significantly increased compared with that in the control rats (Table III). And the ratio of MMP-9/TIMP-1 of longer survival (F2, B2) was significantly lower than that of shorter survival (F1).

Between the bleomycin-induced PF groups, F2 showed a significantly lower MMP-9 concentration and MMP-9/TIMP-1 ratio, and a higher concentration of TIMP-1 compared with the F1 group $(\mathrm{P}<0.05)$. Also, B2 showed a lower MMP-9 and higher MMP-9/TIMP-1 ratio compared with $\mathrm{B} 1(\mathrm{P}<0.05)$.

\section{Discussion}

Bleomycin, as an anticancer drug for a number of human malignancies, is commonly used as a lung fibrosis inducer in bleomycin-rodent animal model (16). In the present study, a Wistar rat model of PF was established by administration a single dose of bleomycin sulfate by intratracheal instillation. A lower body weight increase was observed in the bleomycin-administrated groups without bosentan and the group bosentan treated on the 15 th day compared with that of control rats. This change in the body weight maybe attributed to the progression of the fibrosis (17). When treated with bosentan on the day after bleomycin administration, the rats showed a higher body weight increase compared with the bleomycin induced groups without bosentan treatment, the group with bosentan treated on the 15th day, and a similar body weight increase to the control groups. In addition, no significant difference was detected between the rats treated with bosentanon the 15th day after bleomycin administration and the rats without bosentan treatment after bleomycin administration. Although bosentan was found to inhibit the effect of the bleomycin-induced PF on the body weight of rats, treatment with bosentan at a later time-point resulted in reduced effect. Thus, timely use of bosentan is important to improve the body weight of bleomycin-administrated rats.

Bosentan has been demonstrated to be as effective as dexamethasone for treating lung injury (18). PF is the main side 
A

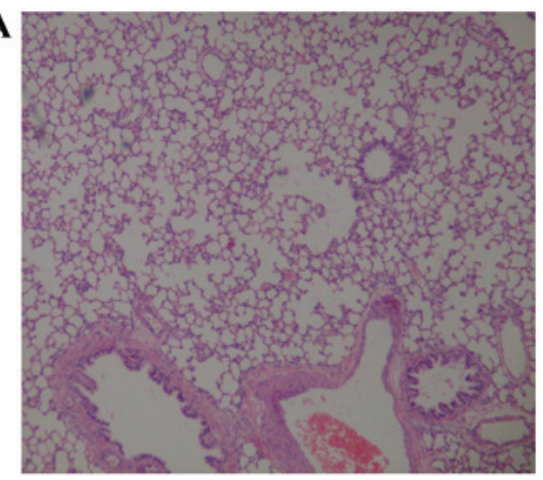

C

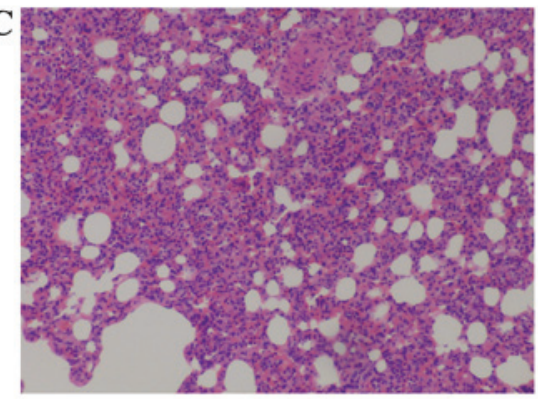

$\mathbf{E}$

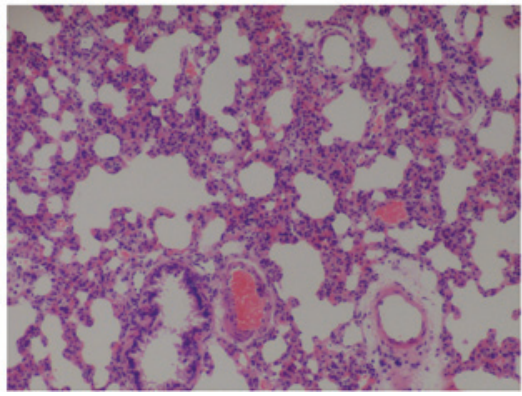

B

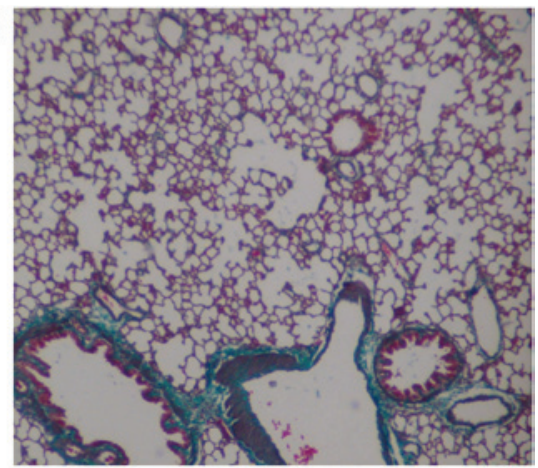

D
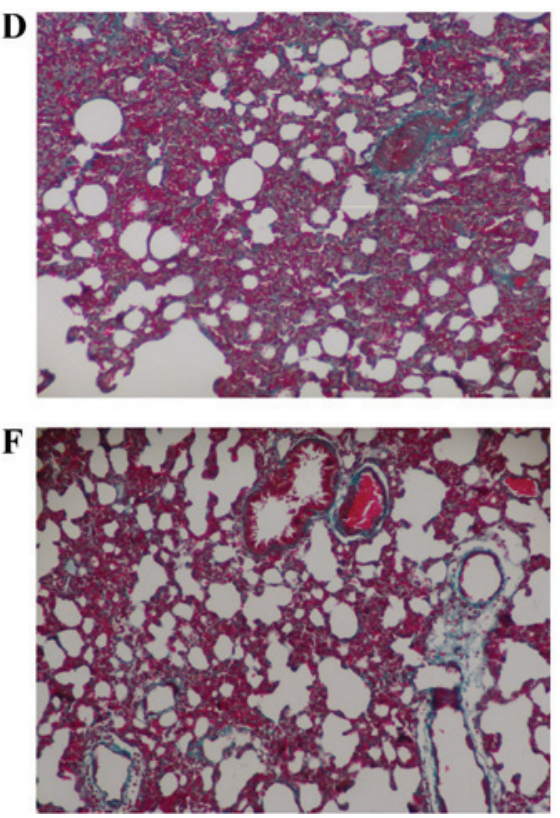

Figure 1. (A) H\&E and (B) Masson's trichrome staining of the control group rat lung tissue samples shows a normal appearance (grade 0). (C) H\&E and (D) Masson's trichrome staining of bleomycin-induced PF group rat lung tissue samples with significant alveolitis and fibrosis. (E) H\&E and (F) Masson's trichrome staining of lung tissue samples from bleomycin-induced PF rats treated with bosentan show reduced alveolitis and fibrosis compared with the bleomycin-induced PF group rats. Magnification, x100. H\&E, hematoxylin and eosin; PF, pulmonary fibrosis.

effect of bleomycin treatment in cancer patients, and the initial damage in the lung leads to inflammatory cell infiltration into the lung parenchyma. The local tissue response to bleomycin-induced lung injury in the present study was evaluated by histopathological examination to confirm the progression of lung fibrosis in bleomycin-administrated rats, as well as to demonstrate the possible protective effect of bosentan against the bleomycin-induced PF. PF is characterized by fibrous hyperplasia and uncontrolled deposition of ECM substances, resulting in destruction of the lung tissue structure (19). It was observed that inflammatory cell infiltration and PF initiation after bleomycin administration in present study. Similar structural changes have been reported in other bleomycin-induced lung fibrosis rat models, thus consolidating the credence of the present experimental model $(17,20-22)$. The results of the current study identified that bleomycin-induced alveolitis and PF were inhibited by bosentan. However, no significant reductions in alveolitis, lung fibrosis and collagen fibers were observed in the group treated with bosentan 15 days after bleomycin administration, which suggested that bosentan was less effective when administered later. Therefore, early treatment with bosentan may reduce alveolitis induced by bleomycin.
It has been reported that fibrosis is a consequence of fibrous hyperplasia subsequent to tissue injury and is known to be closely associated with remodeling of ECM tissue $(23,24)$. PF is characterized by inflammatory cell infiltration, fibroblast differentiation, ECM remodeling and collagen deposition. In addition, MMPs can be produced and released by the epithelium, fibroblasts, myofibroblasts and macrophages, while TIMPs are the inhibitors of MMPs. The activity of MMPs and TIMPs changes with the tissue condition, being low under normal conditions and increasing during certain pathological processes, including repair or tumor development (25). MMP-9, a kind of gelatinases, plays an important role in human and experimental lung fibrosis (26). MMP-9 and TIMPs increase in IPF patients $(27,28)$. Furthermore, it has been reported that MMP-7 serves a critical role in the induction of PF by bleomycin (29). In the present study, bleomycin-treated rats showed reduced levels of MMP-9 and elevated levels of TIMP-1 when compared with the normal control rats. Thus, the bleomycin-treated rats also showed a reduction in the MMP-9/TIMP-1 ratio. These results suggested that the lung injury induced by bleomycin resulted in reduction of MMP-9, elevation of TIMP-1, and the imbalance of MMPs and TIMPs, which are known to promote lung fibrosis 
and result in increased collagen deposition and decreased ECM degradation. Although bosentan failed to affect MMP-9 and TIMP-1 quickly, it decreased the reduction of MMP-9 levels and elevation of TIMP-1 levels. Therefore, timely use of bosentan may help to protect lung tissues against alveolitis and fibrosis induced by bleomycin. As the histopathology of experimental animal models is similarity to PF in humans, bosentan may be suitable as a new drug for the management of PF.

In conclusion, the bleomycin-induced fibrosis model investigated in the present study displayed fibrotic hyperplasia in the lung tissue, as well as reduced MMP-9 and increased TIMP-1 expression. However, bosentan treatment appeared to have a pneumoprotective effect by decreasing in the level of inflammation and fibrous hyperplasia, increasing of MMP-9 expression and reducing TIMP-1 expression. Therefore, bosentan may be used to effectively inhibit PF.

\section{Acknowledgements}

The present study was funded by the Science and Technology Project of Guangdong Province (grant no. 2013B021800045).

\section{References}

1. Selman M, Buendía-Roldán I and Pardo A: Aging and pulmonary fibrosis. Rev Invest Clin 68: 75-83, 2016.

2. Hutchinson J, Fogarty A, Hubbard R and McKeever T: Global incidence and mortality of idiopathic pulmonary fibrosis: A systematic review. Eur Respir J 46: 795-806, 2015.

3. Antoniou KM, Wuyts W, Wijsenbeek M and Wells AU: Medical Therapy in Idiopathic Pulmonary Fibrosis. Semin Respir Crit Care Med 37: 368-377, 2016.

4. Lynch JP III, Huynh RH, Fishbein MC, Saggar R, Belperio JA and Weigt SS: Idiopathic Pulmonary Fibrosis: Epidemiology, Clinical Features, Prognosis, and Management. Semin Respir Crit Care Med 37: 331-357, 2016.

5. Vancheri C, Failla M, Crimi N and Raghu G: Idiopathic pulmonary fibrosis: A disease with similarities and links to cancer biology. Eur Respir J 35: 496-504, 2010.

6. Raghu G, Collard HR, Egan JJ, Martinez FJ, Behr J, Brown KK, Colby TV, Cordier JF, Flaherty KR, Lasky JA, et al; ATS/ERS/JRS/ALAT Committee on Idiopathic Pulmonary Fibrosis: An official ATS/ERS/JRS/ALAT statement: idiopathic pulmonary fibrosis: evidence-based guidelines for diagnosis and management. Am J Respir Crit Care Med 183: 788-824, 2011.

7. Turgut NH, Kara H, Elagoz S, Deveci K, Gungor $\mathrm{H}$ and Arslanbas E: The Protective effect of naringin against bleomycin-induced pulmonary fibrosis in Wistar rats. Pulm Med 2016: 7601393, 2016.

8. Moore BB and Hogaboam CM: Murine models of pulmonary fibrosis. Am J Physiol Lung Cell Mol Physiol 294: L152-L160, 2008.

9. Bonnans C, Chou J and Werb Z: Remodelling the extracellular matrix in development and disease. Nat Rev Mol Cell Biol 15: 786-801, 2014.

10. Zhang Z, Jian X, Zhang W, Wang J and Zhou Q: Using bosentan to treat paraquat poisoning-induced acute lung injury in rats. PLoS One 8: e75943, 2013.

11. Koo HS, Kim KC and Hong YM: Gene expressions of nitric oxide synthase and matrix metalloproteinase-2 in monocrotaline-induced pulmonary hypertension in rats after bosentan treatment. Korean Circ J 41: 83-90, 2011.
12. Giannelli G, Iannone F, Marinosci F, Lapadula G and Antonaci S: The effect of bosentan on matrix metalloproteinase-9 levels in patients with systemic sclerosis-induced pulmonary hypertension. Curr Med Res Opin 21: 327-332, 2005.

13. Wang HD, Yamaya M, Okinaga S, Jia YX, Kamanaka M, Takahashi H, Guo LY, Ohrui T and Sasaki H: Bilirubin ameliorates bleomycin-induced pulmonary fibrosis in rats. Am J Respir Crit Care Med 165: 406-411, 2002.

14. Bancroft JD and Gamble M (eds): Connective tissue stains. In: Theory and practice of histological techniques. 6th edition. Elsevier Health Sciences, Churchill Livingstone, p150, 2008.

15. Szapiel SV, Elson NA, Fulmer JD, Hunninghake GW and Crystal RG: Bleomycin-induced interstitial pulmonary disease in the nude, athymic mouse. Am Rev Respir Dis 120: 893-899, 1979.

16. Sleijfer S: Bleomycin-induced pneumonitis. Chest 120: 617-624, 2001.

17. Zhou XM, Zhang GC, Li JX and Hou J: Inhibitory effects of $\mathrm{Hu}$-qi-yin on the bleomycin-induced pulmonary fibrosis in rats. J Ethnopharmacol 111: 255-264, 2007.

18. Araz O, Demirci E, Yilmazel Ucar E, Calik M, Pulur D, Karaman A, Yayla M, Altun E, Halici Z and Akgun M: Comparison of reducing effect on lung injury of dexamethasone and bosentan in acute lung injury: An experimental study. Multidiscip Respir Med 8: 74-81, 2013.

19. Watson WH, Ritzenthaler JD and Roman J: Lung extracellular matrix and redox regulation. Redox Biol 8: 305-315, 2016.

20. Teixeira KC, Soares FS, Rocha LG, Silveira PC, Silva LA, Valença SS, Dal Pizzol F, Streck EL and Pinho RA: Attenuation of bleomycin-induced lung injury and oxidative stress by $\mathrm{N}$-acetylcysteine plus deferoxamine. Pulm Pharmacol Ther 21: 309-316, 2008

21. Liang X, Tian Q, Wei Z, Liu F, Chen J, Zhao Y, Qu P, Huang X, Zhou X, Liu N, et al: Effect of Feining on bleomycin-induced pulmonary injuries in rats. J Ethnopharmacol 134: 971-976, 2011.

22. Arafa HM, Abdel-Wahab MH, El-Shafeey MF, Badary OA and Hamada FM: Anti-fibrotic effect of meloxicam in a murine lung fibrosis model. Eur J Pharmacol 564: 181-189, 2007.

23. Yoon JS, Chae MK, Jang SY, Lee SY and Lee EJ: Antifibrotic effects of quercetin in primary orbital fibroblasts and orbital fat tissue cultures of Graves' orbitopathy. Invest Ophthalmol Vis Sci 53: 5921-5929, 2012.

24. King TE Jr, Pardo A and Selman M: Idiopathic pulmonary fibrosis. Lancet 378: 1949-1961, 2011.

25. Pardo A, Cabrera S, Maldonado M and Selman M: Role of matrix metalloproteinases in the pathogenesis of idiopathic pulmonary fibrosis. Respir Res 17: 23, 2016.

26. Pardo A and Selman M: Matrix metalloproteases in aberrant fibrotic tissue remodeling. Proc Am Thorac Soc 3: 383-388, 2006.

27. Selman M, Ruiz V, Cabrera S, Segura L, Ramírez R, Barrios R and Pardo A: TIMP-1, $-2,-3$, and -4 in idiopathic pulmonary fibrosis. A prevailing nondegradative lung microenvironment? Am J Physiol Lung Cell Mol Physiol 279: L562-L574, 2000.

28. Ramos C, Montaño M, García-Alvarez J, Ruiz V, Uhal BD, Selman M and Pardo A: Fibroblasts from idiopathic pulmonary fibrosis and normal lungs differ in growth rate, apoptosis, and tissue inhibitor of metalloproteinases expression. Am J Respir Cell Mol Biol 24: 591-598, 2001.

29. Zuo F, Kaminski N, Eugui E, Allard J, Yakhini Z, Ben-Dor A, Lollini L, Morris D, Kim Y, DeLustro B, et al: Gene expression analysis reveals matrilysin as a key regulator of pulmonary fibrosis in mice and humans. Proc Natl Acad Sci USA 99: 6292-6297, 2002. 